\title{
Gravid Uterus Adjusted Maternal Body Weight Gain
}

National Cancer Institute

\section{Source}

National Cancer Institute. Gravid Uterus Adjusted Maternal Body Weight Gain. NCI

Thesaurus. Code C124476.

The amount of maternal body weight gained over a period of time where the weight at the end of the interval is adjusted for the gravid uterus weight. This is derived by subtracting the maternal body weight adjusted for the gravid uterus from the total maternal body weight. 